\title{
Peripheral blood mitochondrial DNA copy number as a novel potential biomarker for diabetic nephropathy in type 2 diabetes patients
}

\author{
GHADA AL-KAFAJI ${ }^{1}$, ABDULAH ALJADAAN ${ }^{1}$, AMER KAMAL ${ }^{2}$ and MOIZ BAKHIET ${ }^{1}$ \\ Departments of ${ }^{1}$ Molecular Medicine and Al-Jawhara Centre for Molecular Medicine, \\ Genetics and Inherited Disorders, and ${ }^{2}$ Physiology, College of Medicine and Medical Sciences, \\ Arabian Gulf University, Manama 329, Kingdom of Bahrain
}

Received March 3, 2018; Accepted May 2, 2018

DOI: $10.3892 /$ etm.2018.6319

\begin{abstract}
The mitochondrial DNA copy number (mtDNA-CN) is a surrogate measure of mitochondrial function and altered mtDNA-CN reflects the oxidant-induced cell damage. A previous study by our group demonstrated that a reduction in the renal mtDNA-CN is implicated in the pathogenesis of diabetic nephropathy (DN), a leading cause of end-stage renal disease in diabetic patients. In the present study, it was investigated whether the mtDNA-CN in the peripheral blood may be utilized as a biomarker for $\mathrm{DN}$ in type 2 diabetes (T2D) patients. The study included 50 non-diabetic and 100 diabetic subjects. The diabetic subjects were sub-divided based on their albumin-to-creatinine ratio (ACR) into T2D patients with normoalbuminuria $(n=50)$, DN patients with microalbuminuria $(n=29)$ and DN patients with macroalbuminuria $(n=21)$. The mtDNA-CN was measured in the peripheral blood by real-time polymerase chain reaction analysis. Patients with DN had a lower mtDNA-CN than patients with T2D and healthy controls $(\mathrm{P}<0.05)$. A sub-group analysis with stratification by the ACR indicated that a decreased mtDNA-CN was associated with the severity and the presence of DN, as it was lower in DN patients with macroalbuminuria than in DN patients with microalbuminuria and T2D patients with normoalbuminuria $(\mathrm{P}<0.01)$. The area under the receiver operating characteristic curve (AUC) for mtDNA-CN was 0.916 (sensitivity, $86 \%$ and specificity, $74 \%$ ) and 0.961 (sensitivity, 96\% and specificity, $88 \%$ ) for differentiating DN patients from T2D patients without DN and from healthy controls, respectively.
\end{abstract}

Correspondence to: Dr Ghada Al-Kafaji, Department of Molecular Medicine and Al-Jawhara Centre for Molecular Medicine, Genetics and Inherited Disorders, College of Medicine and Medical Sciences, Arabian Gulf University, Salmaniya Avenue, Building 293, Road 2904, Block 329, Manama 329, Kingdom of Bahrain

E-mail: ghadaa@agu.edu.bh

Key words: diabetic nephropathy, mitochondrial DNA copy number, peripheral blood, biomarker
Furthermore, the AUC of mtDNA-CN for differentiating DN patients with microalbuminuria from those with macroalbuminuria was 0.895 (sensitivity, $83 \%$ and specificity, $85 \%$ ). Multivariate analysis revealed that the mtDNA-CN was significantly associated with the occurrence and progression of DN, even after adjustment for age, mean blood pressure, glycated haemoglobin A1c and total cholesterol $(\mathrm{P}<0.05)$. In patients with DN, a decreased mtDNA-CN was negatively correlated with albuminuria and conventional risk factors for DN, and was positively correlated with the estimated glomerular filtration rate. The present results therefore suggest the utilization of circulating mtDNA-CN as a novel biomarker for the early diagnosis of DN and indicate the significance of decreased mtDNA-CN as another independent risk factor for DN.

\section{Introduction}

Diabetic nephropathy (DN) is a serious microvascular complication of diabetes mellitus and the leading cause of end-stage renal disease (ESRD). The incidence of DN in type 2 (T2D) patients has noticeably increased with the steady increase in the global prevalence of T2D (1). It is estimated that $40 \%$ of T2D patients develop DN $(2,3)$, and despite advances in therapy and patient care, a large percentage of those patients progress to ESRD (4). The pathogenicity of DN is extremely complex and the molecular mechanisms implicated in the development and progression of $\mathrm{DN}$ remain poorly understood. However, it is well established that hyperglycemia is closely correlated with the progression of $\mathrm{DN}(5,6)$, and increasing evidence indicates that mitochondrial oxidative stress and mitochondrial dysfunction are implicated in disease progression $(7,8)$. Mitochondria are major organelles producing most of the cellular energy in the form of adenosine triphosphate via oxidative phosphorylation, and are the major site of reactive oxygen species (ROS) generation. Overproduction of mitochondrial ROS during hyperglycemia is considered as a unifying mechanism of vascular tissue damage in diabetes $(9,10)$. Indeed, hyperglycemia-induced mitochondrial ROS activate several pathways implicated in DN, including protein kinase $\mathrm{C}$, mitogen-activated protein kinases, as well as various cytokines and transcription factors $(9,10)$, which 
ultimately enhances the expression of extracellular matrix genes with progression to renal fibrosis associated with a declining glomerular filtration rate (GFR) (11).

The pathophysiological basis of DN is the development of persistent microalbuminuria with an urinary albumin excretion rate of 30-300 mg/day, which may progress over a number of years to macroalbuminuria (urinary albumin excretion rate $>300 \mathrm{mg}$ /day), followed by a gradual decline in the GFR and at a later stage to kidney failure (12). Although microalbuminuria has long been considered as the first sign of renal involvement in T2D patients, it lacks the reliability to accurately predict kidney-associated outcomes in diabetic patients $(13,14)$. It has been also indicated that in certain DN patients, renal impairment may occur even prior to the appearance of microalbuminuria, and $\sim 20 \%$ of patients with impaired renal function have normoalbuminuria (15). Therefore, evaluation of different biomarkers and their association with DN at the molecular level may provide a means of early detection and also for the prevention of this progressive disease.

Mitochondria carry their own mitochondrial DNA (mtDNA), a 16.5-kb, double-stranded, circular molecule, which contains genes encoding for proteins that are essential for normal mitochondrial function (16). mtDNA presents in a mammalian cell as a multicopy genome with 1,000-10,000 copies per cell. The quantity of mitochondria is indicated by the mtDNA copy number (mtDNA-CN), which is used as a surrogate measure of mitochondrial function (16). Compared to nuclear DNA (nDNA), mtDNA is more susceptible to oxidative stress due to its close proximity to the site of free radical generation (17). Increased oxidative stress in human cells may contribute to alterations in the mtDNA-CN, mtDNA-encoded gene expression and mitochondrial abundance (18-21). A previous study by our group has indicated that in response to high glucose-induced chronic oxidative stress, the renal mtDNA-CN is decreased and the mitochondria are impaired (21). Furthermore, decreased mtDNA-CN in the peripheral blood has also been reported in several diseases associated with oxidative stress. For instance, low mtDNA-CN in the whole blood of patients with T2D precedes the development of diabetes (22) and a reduction in mtDNA-CN in peripheral leukocytes is associated with insulin resistance in T2D patients (23), insulin sensitivity in offspring of T2D patients (24) and the age at onset of T2D (25). In addition, decreased peripheral blood mtDNA-CN is linked to other diseases, including cancer (26), and associated with the severity of coronary heart disease (27). These clinical and experimental studies suggest that altered mtDNA-CN, which reflects the oxidant-induced cell damage, may provide a useful disease biomarker.

The present study investigated the potential of mtDNA-CN in the peripheral blood as a biomarker for DN. mtDNA-CN was quantified as the DNA ratio between a target mitochondrial gene and a reference nuclear gene $(m t D N A / n D N A)$ in blood samples from T2D patients without DN, T2D patients with DN and non-diabetic healthy subjects.

\section{Materials and methods}

Study population. The study included 50 non-diabetic healthy controls and $100 \mathrm{~T} 2 \mathrm{D}$ patients. Of the T2D patients, 50 had no
DN and 50 had DN. The participants were recruited between November 2013 and January 2015 from King Abdullah University Medical Centre (Arabian Gulf University, Manama, Kingdom of Bahrain). Healthy subjects were recruited during visitation to the Centre either for a routine check-up or due to the fact that they had a family history of T2D.

T2D was diagnosed according to the World Health Organization criteria (28) with fasting glucose (FG) levels of $\geq 7.0 \mathrm{mmol} / \mathrm{l}$ and glycated hemoglobin A1c (HbAlc) levels of $>6.5 \%$.

The albuminuria status was classified based on the albumin-to-creatinine ratio (ACR) as normoalbuminuria (ACR $<2.5 \mathrm{mg} / \mathrm{mmol}$ for men and ACR $<3.5 \mathrm{mg} / \mathrm{mmol}$ for women), microalbuminuria (ACR $2.5-25 \mathrm{mg} / \mathrm{mmol}$ for men and ACR 2.8-28 mg/mmol for women) or macroalbuminuria (ACR of $>25 \mathrm{mg} / \mathrm{mmol}$ for men and ACR $>28 \mathrm{mg} / \mathrm{mmol}$ for women) (29). Renal function was assessed using the estimated glomerular filtration rate (eGFR) based on the Modification of Diet in Renal Disease formula (30). The non-diabetic healthy individuals had no history of diabetes and no evidence of kidney disease. The exclusion criteria were cardiovascular disease, hepatic dysfunction, neoplastic diseases and systemic disorders. Clinical data, including age, gender, body mass index (BMI) and blood pressure, were collected from the medical records of the participants.

Extraction of genomic DNA. Genomic DNA was extracted from peripheral blood samples of $50 \mathrm{~T} 2 \mathrm{D}$ patients, $50 \mathrm{DN}$ patients and 50 non-diabetic healthy subjects using the QIAMP DSP DNA kit (Qiagen, Hilden, Germany) as per the manufacturer's protocols. In brief, $20 \mu 1$ protease was mixed with $200 \mu$ l EDTA-blood followed by the addition of lysis buffer $(200 \mu \mathrm{l})$ and incubation at $56^{\circ} \mathrm{C}$ for $10 \mathrm{~min}$. Following centrifugation at $20,000 \times \mathrm{g}$ for $1 \mathrm{~min}$ at $4^{\circ} \mathrm{C}$, absolute ethanol (200 $\mu \mathrm{l}$ ) was added and the lysate was centrifuged at 6,000 x g for $1 \mathrm{~min}$. Washing steps were performed using $500 \mu \mathrm{l}$ washing buffer followed by centrifugation at 6,000 x $\mathrm{g}$ for $1 \mathrm{~min}$ and then at $20,000 \times \mathrm{g}$ for $3 \mathrm{~min}$. To elute the genomic DNA, elution buffer $(200 \mu 1)$ was added, followed by incubation at room temperature for $1 \mathrm{~min}$ and subsequent centrifugation at $6,000 \times \mathrm{g}$ for $1 \mathrm{~min}$. DNA concentrations were determined using a Nanodrop ND-1000 ultraviolet-visible light spectrophotometer (Thermo Fisher Scientific, Inc., Waltham, MA, USA). The concentrations of the DNA samples were adjusted to $200 \mathrm{ng} / \mu \mathrm{l}$. All genomic DNA samples were stored at $-20^{\circ} \mathrm{C}$ until further analysis.

Determination of mtDNA-CT. Quantitative real-time polymerase chain reaction (qPCR) analysis was used to determine the mtDNA-CN. TaqMan assays designed for the mitochondrial cytochrome b (CYTB) gene and the single-copy nuclear $\beta$-2-microglobulin (B2M) gene were used in the present study. The primers and probes for CYTB and B2M were as follows: CYTB forward primer, 5'-GCCTGCCTGATCCTC CAAAT-3', CYTB reverse primer, 5'-AAGGTAGCGGAT GATTCAGCC-3' and CYTB probe, 5'FAM-CACCAGACG CCTCAACCGCCTT-TAMRA3'; B2M forward primer, 5'-CCAGCAGAGAATGGAAAGTCAA-3', B2M reverse primer, 5'-TCTCTCTCCATTCTTCAGTAAGTCAACT-3' and B2M probe, 5'FAM-ATGTGTCTGGGTTTCATCCAT 
CCGACA-TAMRA3'. The PCR mixture contained 200 nM primers, $100 \mathrm{nM}$ TaqMan probe (Applied Biosystems; Thermo Fisher Scientific, Inc.), 200 ng DNA and 200 nM 2.5 Premix Ex Taq (Takara Bio Inc, Otsu, Japan) in a final volume of $20 \mu$ l. qPCR was performed with a 7900HT real-time PCR system (Applied Biosystems; Thermo Fisher Scientific, Inc.) using the following cycling conditions: 1 cycle of $50^{\circ} \mathrm{C}$ for $2 \mathrm{~min}, 1$ cycle of $95^{\circ} \mathrm{C}$ for $10 \mathrm{~min}$, and 30 cycles of $95^{\circ} \mathrm{C}$ for $15 \mathrm{sec}$ and $60^{\circ} \mathrm{C}$ for $1 \mathrm{~min}$. For each PCR run, standard curves were established from 5 serially diluted, purified PCR products $\left(1 \times 10^{2}\right.$ to $\left.1 \times 10^{7} \mathrm{ng} / \mathrm{ml}\right)$ from the CYTB gene and the B2M gene to generate quantification data as previously described (19,21). Quantification of mtDNA was accomplished by calculating the ratio of mtDNA-encoded CYTB gene to the nDNA-encoded B2M gene and mtDNA-CN data were expressed as mtDNA per nDNA. Each measurement was repeated twice and a non-template control (with template DNA omitted) was included in each experiment.

Statistical analysis. SPSS software (version 23; IBM Corp., Armonk, NY, USA) was used for statistical analysis. A two-tailed Student's t-test was used for comparison between two groups and one-way analysis of variance followed by a post hoc Tukey's HSD test for multiple-group comparisons. The receiver operating characteristic (ROC) curve was used as a measure of diagnostic accuracy of mtDNA-CN and the area under the curve (AUC) was calculated to evaluate the specificity and sensitivity. The association between mtDNA-CN and DN was assessed using multivariate regression models. The odds ratios (ORs) and 95\% confidence intervals (CIs) were computed with these models with multi-adjustment for clinical variables. The correlation between mtDNA-CN and clinical variables was analyzed by calculating Pearson's correlation coefficient. $\mathrm{P}<0.05$ was considered to indicate a statistically significant difference. All values are expressed as the mean \pm standard deviation.

\section{Results}

Clinical data. A total of 50 non-diabetic healthy subjects and 100 T2D patients were included in the present study. Of all of the patients, 50 patients with T2D had DN and 50 had no DN. The diabetic subjects were sub-divided based on the ACR into T2D patients with normoalbuminuria $(n=50), D N$ patients with microalbuminuria $(n=29)$ and DN patients with macroalbuminuria $(n=21)$. The clinical data of the participants are displayed in Table I. The patients with T2D with and without $\mathrm{DN}$ were older than the non-diabetic individuals $(\mathrm{P}<0.05)$. However, there was no significant difference in gender between the controls and the patients with T2D with and without DN ( $\mathrm{P}>0.05)$. Other parameters, including the BMI, mean blood pressure, FG, HbA1c, albuminuria, ACR, serum creatinine, estimated (e)GFR, low-density lipoprotein (LDL) and triglycerides differed significantly between the controls and the patients with T2D with and without DN $(\mathrm{P}<0.05)$. Furthermore, the T2D patients with $\mathrm{DN}$ were older than those without $\mathrm{DN}(\mathrm{P}=0.012)$ and had a significantly higher blood pressure $(\mathrm{P}<0.05)$, albuminuria $(\mathrm{P}<0.05)$, ACR $(\mathrm{P}<0.05)$, serum creatinine $(\mathrm{P}<0.05)$, LDL $(\mathrm{P}<0.05)$ and triglycerides $(\mathrm{P}<0.05)$, and a lower eGFR $(\mathrm{P}<0.05)$. In addition, $\mathrm{DN}$ patients with macroalbuminuria were older, had a higher mean blood pressure and a longer duration of diabetes than those with microalbuminuria and T2D patients with normoalbuminuria $(\mathrm{P}<0.05)$. Patients with macroalbuminuria also had higher levels of FG, HbAlc, albuminuria, ACR, serum creatinine, LDL, triglycerides, total cholesterol and lower eGFR than patients with microalbuminuria and normoalbuminuria $(\mathrm{P}<0.05)$. In terms of sex, there were more males in the microalbuminuria group than in the macroalbuminuria group $(\mathrm{P}<0.05)$.

Accuracy of qPCR assays. For accurate quantification of mtDNA-CN, standard curves were established using dilution series of purified PCR products obtained from the mtDNA gene CYTB and the nDNA gene B2M as previously described $(19,21)$. The regression curves calculated with five dilutions ranging from $1 \times 10^{2}$ to $1 \times 10^{7} \mathrm{ng} / \mathrm{ml}$ had excellent and significant correlation coefficients $(\mathrm{r}-1.00, \mathrm{P}=0.013$ for $\mathrm{CYTB}$ and $\mathrm{r}-1.00, \mathrm{P}=0.024$ ) for $\mathrm{B} 2 \mathrm{M}$ ), indicating a high accuracy of mtDNA-CN quantification.

Peripheral blood mtDNA-CN is decreased in T2D patients without and further decreased in those with DN. The mtDNA-CN, defined as the copy number ratio of mtDNA to nDNA (CYTB/B2M) was measured in peripheral blood samples from T2D patients without DN, T2D patients with DN and non-diabetic healthy controls by TaqMan-based real-time PCR.

As presented in Fig. 1, T2D patients with DN had a significantly lower mtDNA-CN than those without DN and the healthy control subjects $(\mathrm{P}<0.01)$. Furthermore, the mtDNA-CN was significantly lower in T2D patients than in healthy controls $(\mathrm{P}=0.048)$. The mean mtDNA copy number was $39.75 \pm 6.6$ in healthy subjects, $35.11 \pm 5.4$ in T2D patients and $20.85 \pm 5.2$ in DN patients.

Peripheral blood mtDNA-CN is decreased with the severity of $D N$. Next, the association between peripheral blood mtDNA-CN and the severity of DN was examined by measurement of albuminuria in the patient groups. Based on the ACR, the patients were categorized into T2D patients without DN and normoalbuminuria $(\mathrm{n}=50)$, and T2D patients with $\mathrm{DN}$ and microalbuminuria $(n=29)$ or macroalbuminuria $(n=21)$.

The sub-group analysis with stratification based on the ACR indicated that the mtDNA-CN in patients with macroalbuminuria was significantly lower than that in patients with microalbuminuria and normoalbuminuria $(\mathrm{P}<0.01$; Fig. 2$)$. Of note, the mtDNA-CN in patients with macroalbuminuria was also significantly lower than that in patients with microalbuminuria $(\mathrm{P}<0.01)$. The mean mtDNA-CN was $35.11 \pm 5.4$ in normoalbuminuria patients, $23.8 \pm 3.5$ in microalbuminuria patients and $16.7 \pm 2.9$ in macroalbumiuria patients.

ROC analysis of peripheral blood $m t D N A-C N$. Analysis using the ROC curve was used to evaluate the diagnostic value of peripheral blood mtDNA-CN as a biomarker for DN.

The results revealed that the AUC of mtDNA-CN for discriminating T2D patients with DN from those without DN was 0.92 (95\% CI: 0.86-0.97, P<0.01; Fig. 3A) with a sensitivity of $86 \%$ and a specificity of $74 \%$ (Table II). Furthermore, regarding the discrimination of $\mathrm{T} 2 \mathrm{D}$ patients with $\mathrm{DN}$ from 


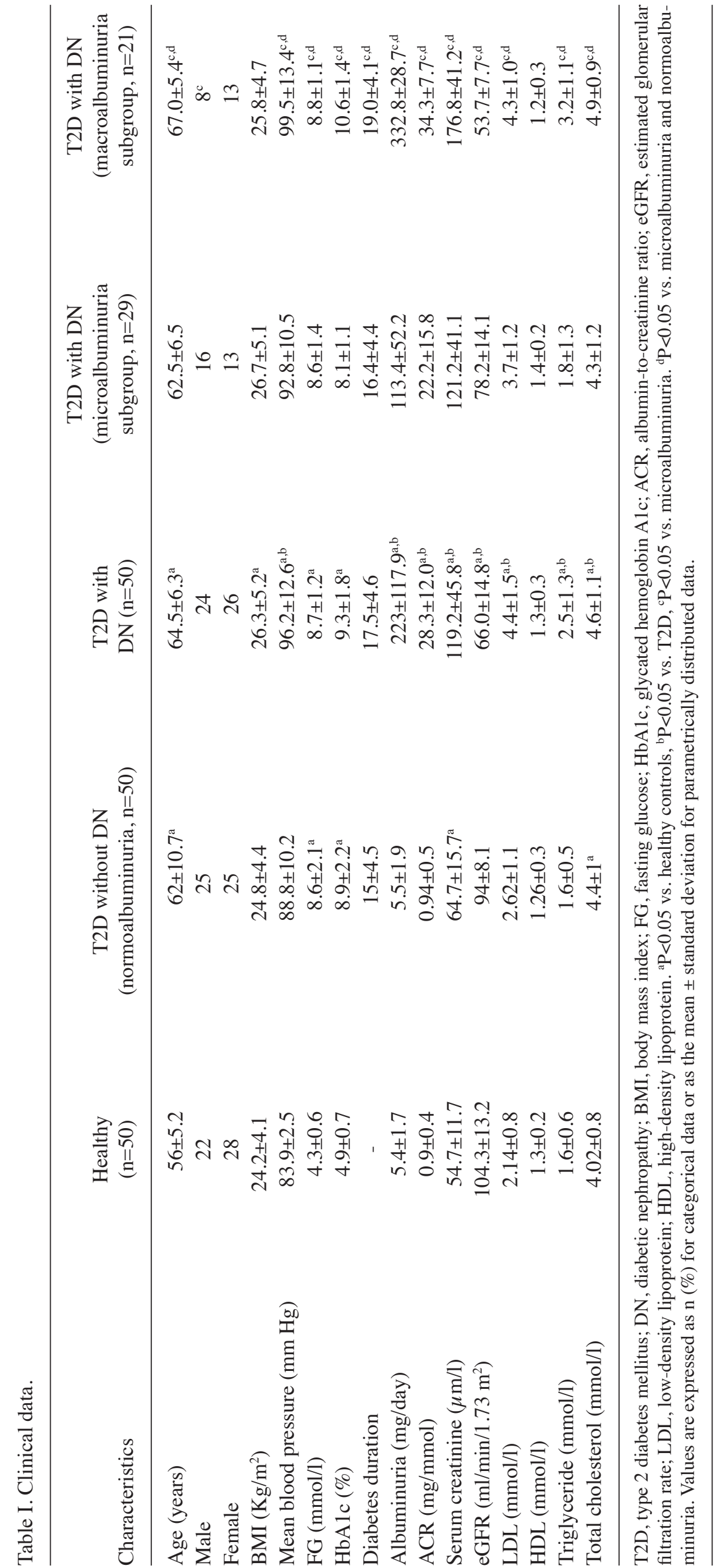




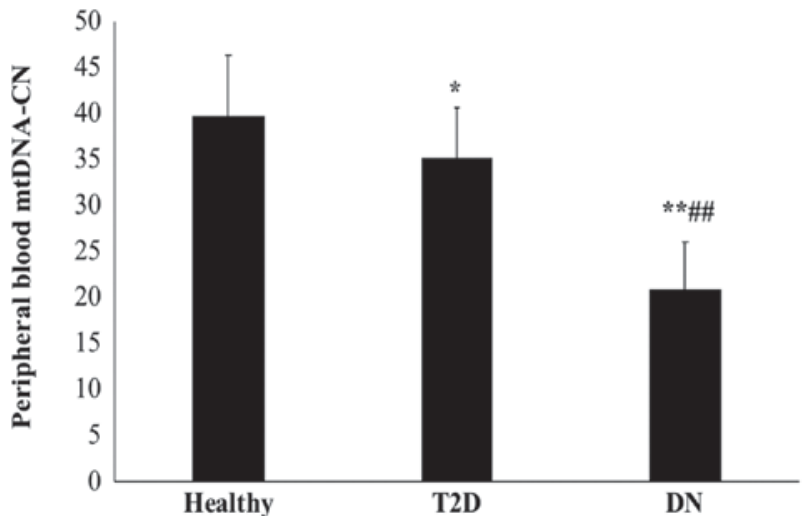

Figure 1. Peripheral blood mtDNA-CN in T2D patients without and with DN and in healthy subjects. Peripheral blood mtDNA-CN was measured in T2D patients without and with $\mathrm{DN}$, as well as in non-diabetic healthy subjects by TaqMan-based real time polymerase chain reaction analysis using standard curves for the mtDNA gene CYTB and the nuclear gene B2M, and expressed as the CYTB/B2M ratio. ${ }^{*} \mathrm{P}<0.05$ and ${ }^{* *} \mathrm{P}<0.01$ vs. healthy controls; ${ }^{\# \#} \mathrm{P}<0.01$ vs. T2D. DN, diabetic nephropathy; T2D, type 2 diabetes mellitus; CYTB, mitochondrial cytochrome b; B2M, $\beta$-2-microglobulin; mtDNA-CN, mitochondrial DNA copy number.

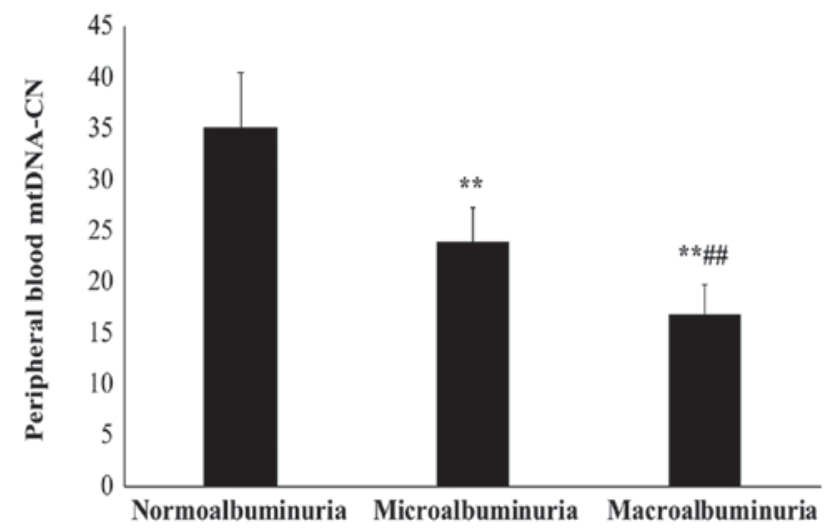

Figure 2. Peripheral blood mtDNA-CN is decreased with the severity of DN. The association between mtDNA-CN and the severity of DN was analyzed in the patient groups stratified based on albumin-to-creatinine ratio into type 2 diabetes mellitus patients with normoalbuminuria $(\mathrm{n}=50)$, DN patients with microalbuminuria $(n=29)$ and $D N$ patients with macroalbumiuria $(n=21)$ ${ }^{* * *} \mathrm{P}<0.01$ compared with normoalbuminuria and ${ }^{\# \#} \mathrm{P}<0.01$ compared with microalbuminuria. mtDNA-CN, mitochondrial DNA copy number; DN, diabetic nephropathy.

healthy controls, the AUC for mtDNA-CN was 0.96 (95\% CI: $0.92-1.000, \mathrm{P}<0.01$; Fig. 3B) with a sensitivity of $96 \%$ and a specificity of $88 \%$ (Table II). The AUC of mtDNA-CN for differentiating between patients with microalbuminuria and macroalbuminuria was 0.895 (95\% CI: $0.81-0.98, \mathrm{P}<0.01$; Fig. 3C) with a sensitivity of $83 \%$ and a specificity of $85 \%$ (Table II). The sensitivity and specificity of a quantitative test were dependent on the cut-off points above or below which the test is positive. The higher the sensitivity, the lower the specificity and vice versa. The optimal cut-off values were selected from the maximum sensitivity and specificity values of the ROC curve output.

Association between peripheral blood mtDNA-CN and DN. A multivariate logistic regression analysis was performed
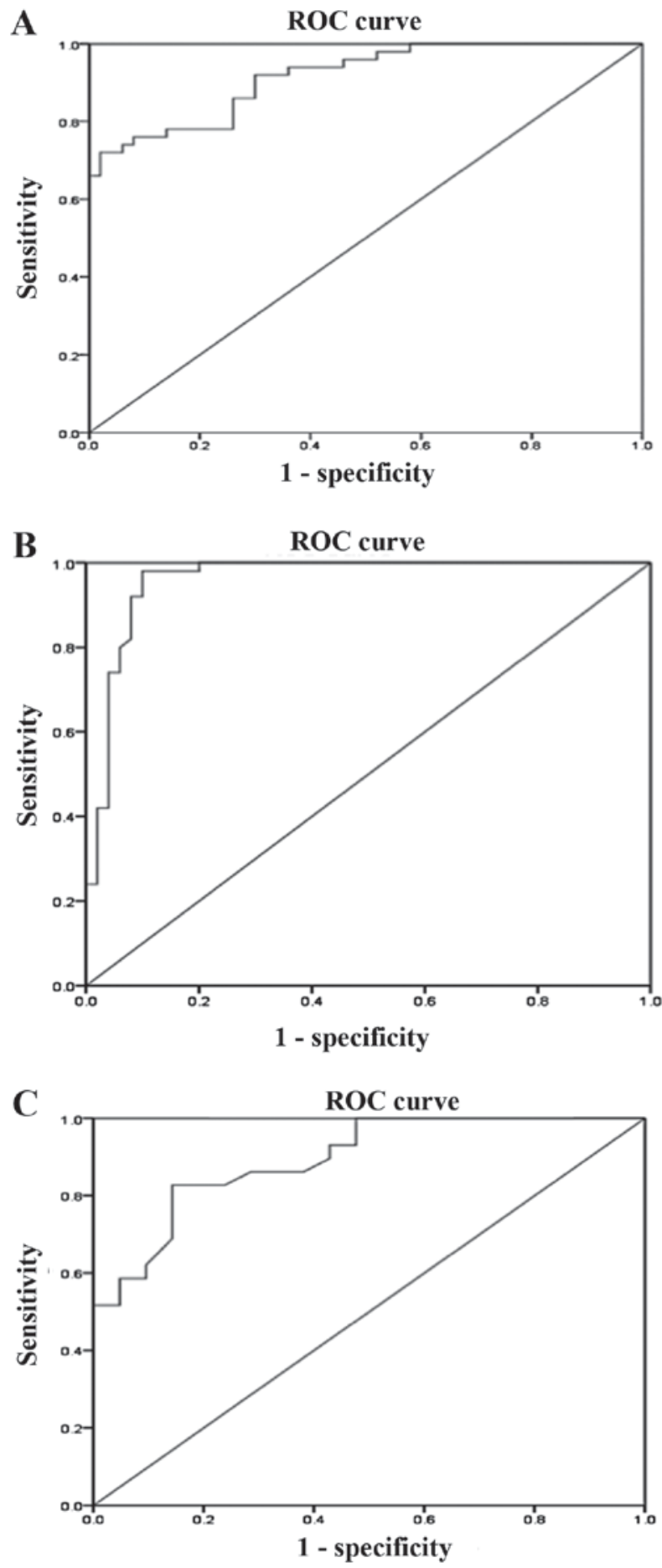

Figure 3. ROC analysis for mtDNA-CN. ROC curves were generated to evaluate the diagnostic value of peripheral blood mtDNA-CN as a biomarker for DN. (A) The AUC for the differentiation between T2D patients with and without DN based on their mtDNA-CN was 0.92 (95\% CI: 0.86-0.97, $\mathrm{P}<0.01$ ). (B) mtDNA-CN levels were suitable for differentiating between $\mathrm{DN}$ patients and healthy controls with an AUC of 0.96 (95\% CI: 0.92-1.000, P<0.01). (C) The AUC for the differentiation of T2D patients with DN and microalbuminuria from those with macroalbuminuria based on their mtDNA-CN was 0.895 (95\% CI: $0.81-0.98, \mathrm{P}<0.01)$. ROC, receiver operating characteristic; AUC, area under the curve; $\mathrm{CI}$, confidence interval; mtDNA-CN, mitochondrial DNA copy number; DN, diabetic nephropathy; T2D, type 2 diabetes.

using different models to define the association between peripheral blood mtDNA-CN and DN. The group of patients with T2D without DN was used as the reference category and 
Table II. Receiver operating characteristic analysis for the peripheral blood mitochondrial DNA copy number.

\begin{tabular}{lcccccc}
\hline Comparison of groups & AUC & 95\% CI & P-value & Cut-off point & Sensitivity (\%) & Specificity (\%) \\
\hline DN vs. T2D & 0.916 & $0.864-0.968$ & $<0.01$ & 24.75 & 86 & 74 \\
DN vs. healthy & 0.961 & $0.921-1.000$ & $<0.01$ & 28.95 & 96 & 88 \\
Microalbuminuria vs. macroalbuminuria & 0.895 & $0.810-0.980$ & $<0.01$ & 21.33 & 83 & 85 \\
\hline
\end{tabular}

T2D, type 2 diabetes; DN, diabetic nephropathy; AUC, area under the curve; CI, confidence interval.

Table III. Association between the peripheral blood mtDNA-CN and DN.

\begin{tabular}{|c|c|c|c|c|c|c|}
\hline \multirow[b]{3}{*}{ Models } & \multicolumn{3}{|c|}{ The reference category: T2D group without DN } & \multicolumn{3}{|c|}{ The reference category: Healthy control group } \\
\hline & \multicolumn{3}{|c|}{ DN } & \multicolumn{3}{|c|}{ DN } \\
\hline & OR & $95 \% \mathrm{CI}$ & P-value & OR & $95 \% \mathrm{CI}$ & P-value \\
\hline Model 1 & 0.662 & $0.552-0.792$ & $<0.01$ & 0.694 & $0.593-0.812$ & $<0.01$ \\
\hline Model 2 & 0.712 & $0.600-0.845$ & $<0.01$ & 0.696 & $0.594-0.85$ & 0.015 \\
\hline Model 3 & 0.662 & $0.516-0.851$ & $<0.01$ & 0.698 & $0.592-0.833$ & 0.02 \\
\hline Model 4 & 0.634 & $0.474-0.848$ & 0.028 & 0.671 & $0.558-0.806$ & $<0.01$ \\
\hline
\end{tabular}

The reference category: T2D without DN (nornoalbuminuria group)

\begin{tabular}{lccccccc}
\hline & \multicolumn{3}{c}{ DN with microalbuminuria } & & \multicolumn{3}{c}{ DN with macroalbuminuria } \\
\cline { 2 - 3 } Models & OR & $95 \% \mathrm{CI}$ & P-value & & OR & $95 \%$ CI & P-value \\
\hline Model 1 & 0.727 & $0.620-0.853$ & $<0.01$ & & 0.463 & $0.345-0.621$ & $<0.01$ \\
Model 2 & 0.728 & $0.620-0.855$ & $<0.01$ & & 0.461 & $0.340-0.625$ & $<0.01$ \\
Model 3 & 0.718 & $0.608-0.848$ & $<0.01$ & & 0.443 & $0.316-0.622$ & $<0.01$ \\
Model 4 & 0.697 & $0.579-0.839$ & 0.047 & & 0.480 & $0.336-0.684$ & 0.017 \\
\hline
\end{tabular}

T2D, type 2 diabetes; DN, diabetic nephropathy; OR, odds ratio; CI, confidence interval. Model 1, unadjusted. Model 2, adjusted for age. Model 3, adjusted for age and mean blood pressure. Model 4 adjusted for age, mean blood pressure, HbA1c, and total cholesterol.

the group of T2D patients with DN as the dependent variable. The results indicated a significant association between mtDNA-CN and the occurrence of DN ( $\mathrm{P}<0.05$; Table III). This association remained significant after adjustment for several variables, including age, mean blood pressure, HbA1c and total cholesterol $(\mathrm{P}<0.05)$. In addition, when the group of non-diabetic healthy control subjects was regarded as the reference category and the group of DN patients as the dependent variable (Table III), the mtDNA-CN was significantly associated with the presence of DN prior to and after adjustment for the abovementioned clinical variables $(\mathrm{P}<0.05)$.

Next, the normoalbuminuria group (T2D patients without DN) was regarded as the reference category and the microalbuminuria or macroalbuminuria group as the dependent variable (Table III). It was revealed that the mtDNA-CN was independently associated with microalbuminuria and macroalbuminuria even after adjustment for age, mean blood pressure, HbAlc and total cholesterol $(\mathrm{P}<0.05)$, suggesting its independent association with the progression of $\mathrm{DN}$.

Correlation analysis. Pearson's correlation coefficient was calculated to determine the correlation between peripheral blood mtDNA-CN and different clinical variables in T2D patients with DN.

Regarding the kidney function parameters, the results indicated that mtDNA-CN was negatively correlated with the ACR ( $r=-0.66, P=0.02$; Fig. 4A) and urinary albumin excretion (UAE; $\mathrm{r}=-0.63, \mathrm{P}<0.05$; Fig. 4B), and was positively correlated with the eGFR ( $\mathrm{r}=0.69, \mathrm{P}<0.05$; Fig. 4C).

Regarding other clinical variables (Table IV), the mtDNA-CN was negatively correlated with age $(r=-0.32$, $\mathrm{P}<0.05)$, mean blood pressure $(\mathrm{r}=-0.22, \mathrm{P}=0.021), \mathrm{FG}(\mathrm{r}=-0.25$, $\mathrm{P}<0.05), \mathrm{HbA} 1 \mathrm{c}(\mathrm{r}=-0.50, \mathrm{P}<0.05)$ and diabetes duration $(\mathrm{r}=-0.44, \mathrm{P}<0.05)$, but was positively correlated with the BMI $(\mathrm{r}=0.06, \mathrm{P}<0.005)$. In addition, the mtDNA-CN was negatively 
Table IV. Correlation between peripheral blood mtDNA-CN and clinical parameters of T2D patients with DN.

\begin{tabular}{lcc}
\hline Parameter & $\mathrm{r}$ & P-value \\
\hline Age & -0.32 & $<0.01$ \\
BMI & 0.06 & $<0.01$ \\
Mean blood pressure & -0.22 & 0.021 \\
FG & -0.25 & $<0.01$ \\
HbA1c & -0.50 & $<0.01$ \\
Diabetes duration & -0.44 & $<0.01$ \\
LDL & -0.31 & 0.034 \\
Triglycerides & -0.53 & $<0.01$ \\
Total cholesterol & -0.34 & $<0.01$ \\
\hline
\end{tabular}

T2D, type 2 diabetes; DN, diabetic nephropathy; $r$, Pearson's correlation coefficient; BMI, body mass index; FG, fasting glucose; HbA1c, glycated hemoglobin A1c; LDL, low-density lipoprotein.

correlated with various lipid profile parameters, including LDL $(\mathrm{r}=-0.31, \mathrm{P}=0.034)$, triglycerides $(\mathrm{r}=-0.53, \mathrm{P}<0.05)$ and total cholesterol $(\mathrm{r}=-0.34, \mathrm{P}<0.05)$.

\section{Discussion}

The earliest clinical indication of diabetic nephropathy (DN) is microalbuminuria, which may progress over a number of years (4-6 years) to macroalbuminuria (12). This results in a gradual decline in glomerular filtration rate, eventually leading to end stage renal disease (12). However, renal impairment may occur prior to microalbuminuria in certain patients with DN (15). Furthermore, $20 \%$ of patients with impaired renal function exhibit normoalbuminuria (15). In the present study, T2D patients without DN (with normoalbuminuria), DN patients with microalbuminuria or macroalbuminuria, and non-diabetic healthy controls were selected to investigate the potential of the mtDNA-CN as a marker for DN. The mtDNA-CN was measured by qPCR in the presence of calibration standards as the ratio between a target mitochondrial gene and a reference nuclear gene (CYTB vs. B2M) in blood samples from the subject groups.

Using qPCR, it was identified that the mtDNA-CN was significantly decreased in the peripheral blood of DN patients compared with that in T2D patients without DN and the controls. Furthermore, the mtDNA-CN was progressively declined in patients with higher levels of albuminuria, and was lower in the macroalbuminuric group than in the microalbuminuric and normoalbuminuric groups, suggesting an association between mtDNA-CN and the severity of DN.

Consistent with the results of decreased peripheral blood mtDNA-CN in the T2D patients of the present study, Lee et al (22) determined low mtDNA-CN in pre-diabetic patients who developed T2D after two years. Other studies also indicated a decline in peripheral blood mtDNA-CN in association with insulin resistance and insulin sensitivity $(23,24)$, as well as with the age at onset of T2D (25). Decreased peripheral blood mtDNA-CN was also reported in other diseases
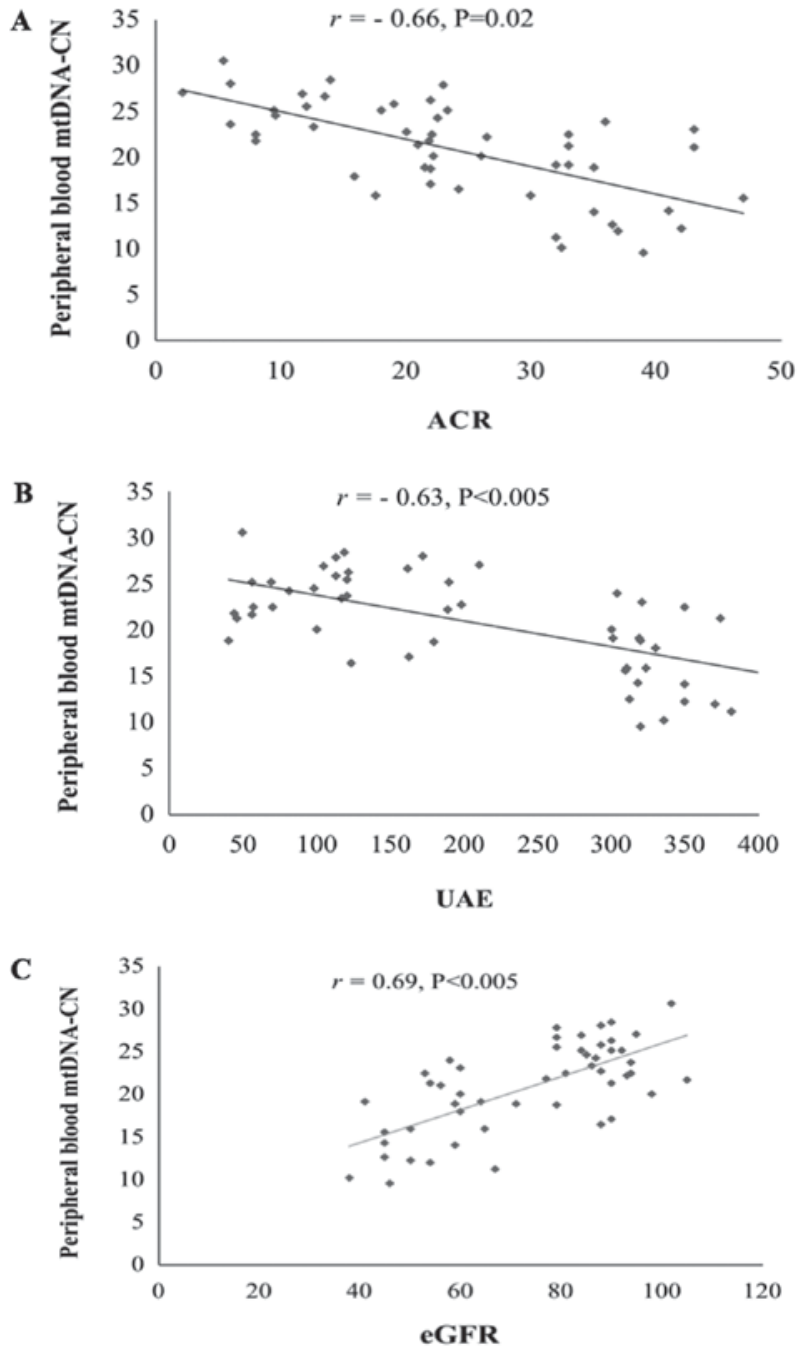

Figure 4. Correlation between peripheral blood mtDNA-CN and kidney function parameters. Pearson's correlation analysis was performed to determine the correlation between mtDNA-CN and kidney function parameters in T2D patients with DN. (A) Correlation between mtDNA-CN and ACR. (B) Correlation between mtDNA-CN and UAE. (C) Correlation between mtDNA-CN and eGFR. ACR, albumin-to-creatinine ratio; mtDNA-CN, mitochondrial DNA copy number; DN, diabetic nephropathy; eGFR, estimated glomerular filtration rate; UAE, urinary albumin excretion.

associated with oxidative stress, including cancer and cardiovascular disease $(26,27)$.

While a previous study by Lee et al (31) demonstrated an association between mtDNA-CN in the peripheral blood and the prevalence of microalbuminuria in patients with chronic kidney disease, the present study was the first to investigate the peripheral blood mtDNA-CN in T2D patients with different levels of albuminuria, in patients with T2D without DN and including normoalbuminuria, and patients with DN with microalbuminuria and macroalbuminuria and its link with the severity of DN.

Mitochondria, the major intracellular source of energy and the major site of ROS generation, have their own DNA, which encodes genes for proteins that are essential for normal mitochondrial function (16). The copy number of mtDNA reflects the abundance of mitochondria and may change according to the cell's energy requirements, as well as the physiological or environmental conditions $(16,18)$. Due to its close proximity to 
the sites of free radical generation in the mitochondria, mtDNA is particularly vulnerable to oxidative damage (17). The oxidative damage to mtDNA may impair the electron transport system and results in a decline in mitochondrial function, which in turn leads to enhanced ROS production and further oxidative damage of mtDNA $(17,32)$. Mitochondrial dysfunction is central to the pathogenesis of diabetes and its complications that include DN $(7,8)$. According to the conventional theory, hyperglycemia-induced overproduction of mitochondrial ROS has a major role in diabetic vascular complications $(9,10)$. In vivo and in vitro studies have indeed demonstrated that elevated ROS production and increased oxidative damage of mtDNA are linked to the pathogenicity and development of DN (33-35). Increased oxidative stress may have a critical role in regulating the mtDNA-CN in stressed cells (18). Although the mtDNA-CN may be increased in response to initial oxidative stress to compensate for damaged DNA $(18,19)$, chronic oxidative stress decreases the mtDNA-CN and increases mtDNA damage $(18,21)$. A previous study by our group identified a significant decrease in renal mtDNA-CN and mitochondrial function in response to high glucose-induced chronic oxidative stress (21). In the present study, decreased peripheral blood mtDNA-CN was identified in DN patients, which was correlated with the development DN. These results suggest that decreased mtDNA-CN in the peripheral blood of DN patients may be a consequence of diabetes-induced oxidative stress. In support of this notion, previous studies have indicated that diabetic patients with DN have higher oxidative stress than non-diabetic individuals $(36,37)$. Furthermore, a study by Zhou et al (38) reported that reduced peripheral blood mtDNA-CN as a result of hyperglycemia-induced oxidative stress is closely associated with glucose-stimulated insulin secretion in diabetic patients.

In recent years, activation of the immune system and chronic inflammation have been proposed as novel pathways involved in DN. Various inflammatory molecules, including chemokines, adhesion molecules, pro-inflammatory cytokines and growth factors, nuclear factors, as well as immune cells, including monocytes, lymphocytes and macrophages, have been reported to have important roles in DN (39). Altered mitochondrial biology has been implicated in the development of chronic systemic inflammation and impaired physiological function (40). Inflammation-induced mitochondrial dysfunction results in decreased oxidative phosphorylation and increased oxidative stress (41). Furthermore, low mtDNA-CN has been indicated to be linked with inflammation in aged individuals (42).

For the study of mtDNA-CN, DNA is usually obtained from peripheral blood mononuclear cells (PBMCs). However, platelet contamination may lead to overestimation of mtDNA-CN measurements in such samples (43). In addition, it has been indicated that healthy individuals have more platelets (14-90 times higher) than leukocytes in peripheral blood, and this may affect the quantification of mtDNA-CN (44). It has been recently reported that not taking the platelet/leukocyte ratio into account in whole-blood measurements may lead to overestimation and misclassification if interpreted as leukocyte mtDNA-CN (44). Therefore, most large-scale epidemiological studies on genetic factors for common diseases have used DNA from whole blood and not from PBMCs (45). Several studies have also used whole blood to measure mtDNA-CN in variety of diseases, including T2D (22) and cancer (46).
The present study evaluated the diagnostic value of peripheral blood mtDNA-CN in DN using ROC analysis. The mtDNA-CN was revealed to have an excellent value in determining $\mathrm{DN}$, as demonstrated by its ability to discriminate DN patients from T2D patients and from healthy subjects with high sensitivity and specificity. In addition, the mtDNA-CN displayed an excellent ability in separating patients with microalbuminuria and macroalbuminuria with good sensitivity and specificity.

Patients with T2D may remain undiagnosed for numerous years and chronic diabetic complications may already be present at the time of diagnosis $(47,48)$. As $\sim 40 \%$ of T2D patients are at risk of developing DN $(2,3)$, biomarkers for the early detection of DN are essential for the timely management of the disease prior to its progression. Although microalbuminuria has long been used as a clinical indicator of DN, the test lacks specificity and accuracy as a predictive marker (13-15). The present results clearly demonstrated and provided the first evidence that the mtDNA-CN may serve as a novel blood biomarker for DN.

Previous studies have reported the potential of mtDNA-CN in the peripheral blood as a diagnostic biomarker for T2D (49), and as a marker to predict the clinical outcomes of patients with ESRD (50). A recent study by Mishra et al (51) also suggested that a decreased mtDNA-CN in the peripheral blood is a potential biomarker for diabetic retinopathy.

The results of the present study further confirmed the association between the mtDNA-CN and DN. As demonstrated by multivariate logistic regression analysis, the mtDNA-CN was significantly and independently associated with the occurrence and the development of DN even after adjustment for several variables, including age, mean blood pressure, HbAlc and total cholesterol. As hyperglycemia, hypertension and hyperlipidemia are well-established risk factors for DN progression $(52,53)$, the present results indicate that decreased mtDNA-CN in DN patients is an independent risk factor for DN.

In the Pearson's correlation coefficient analysis performed on T2D patients with DN in the present study, the mtDNA-CN was identified to be negatively correlated with kidney function parameters, including ACR, UAE rate and serum creatinine, and positively correlated with the eGFR. In addition, the mtDNA-CN was negatively correlated with age, mean blood pressure, glycemic parameters (FG, HbAlc and diabetes duration) and lipid parameters (LDL, triglycerides and total cholesterol). These results suggest an association of decreased mtDNA-CN with the decline in renal function and the progression of DN.

In conclusion, the results of the present study suggest that mtDNA-CN may serve as a novel blood biomarker for the early diagnosis of $\mathrm{DN}$ and that decreased mtDNA-CN is another risk factor for DN. These results may be of importance in the aspect of preventive medicine, and further validation in a larger cohort is required.

\section{Acknowledgements}

The authors would like to thank the technical staff at the Department of Molecular Medicine and the Al-Jawhara Centre for Molecular Medicine, Genetics and Inherited Disorders, 
College of Medicine and Medical Sciences, (Arabian Gulf University, Kingdom of Bahrain) for their assistance.

\section{Funding}

This work was supported by the College of Medicine and Medical Sciences, Arabian Gulf University, Kingdom of Bahrain (grant no. 81).

\section{Availability of data and materials}

The datasets used and/or analyzed during the current study are available from the corresponding author on reasonable request.

\section{Authors' contributions}

GA developed the project and edited the manuscript; AA, AK and MB collected the data; and all authors performed data analysis, managed the data and wrote the manuscript. The final version of the manuscript has been read and approved by all authors, and each author believes that the manuscript represents honest work.

\section{Ethical approval and consent to participate}

All subjects provided written informed consent. The study received ethical approval from the Research and Ethics Committee of the College of Medicine and Medical Sciences (Arabian Gulf University, Manama, Kingdom of Bahrain).

\section{Consent for publication}

Not applicable.

\section{Competing interests}

The authors declare that they have no competing interests.

\section{References}

1. Wild S, Roglic G, Green A, Sicree R and King H: Global prevalence of diabetes: Estimates for the year 2000 and projections for 2030. Diabetes Care 27: 1047-1053, 2004

2. Ghaderian SB, Hayati F, Shayanpour S and Mousavi SSB Diabetes and end-stage renal disease; a review article on new concepts. J Renal Inj Prev 4: 28-33, 2015.

3. Ritz E and Orth SR: Nephropathy in patients with type 2 diabetes mellitus. N Engl J Med 341: 1127-1133, 1999.

4. Remuzzi G and Ruggenenti P: Slowing the progression of diabetic nephropathy. N Engl J Med 329: 1496-1497, 1993.

5. Nathan DM, Bayless M, Cleary P, Genuth S, Gubitosi-Klug R, Lachin JM, Lorenzi G and Zinman B; DCCT/EDIC Research Group: Diabetes control and complications trial/epidemiology of diabetes interventions and complications study at 30 years: Advances and contributions. Diabetes 62: 3976-3985, 2013.

6. Implications of the diabetes control and complications trial. American diabetes association. Diabetes 42: 1555-1558, 1993.

7. Forbes JM, Coughlan MT and Cooper ME: Oxidative stress as a major culprit in kidney disease in diabetes. Diabetes 57: 1446-1454, 2008.

8. Sivitz WI and Yorek MA: Mitochondrial dysfunction in diabetes: From molecular mechanisms to functional significance and therapeutic opportunities. Antioxid Redox Signal 12: 537-577, 2010 .
9. Nishikawa T, Edelstein D, Du X L, Yamagishi S, Matsumura T, Kaneda Y, Yorek MA, Beebe D, Oates PJ, Hammes HP, et al: Normalizing mitochondrial superoxide production blocks three pathways of hyperglycaemic damage. Nature 404: 787-790, 2000.

10. Brownlee M: Biochemistry and molecular cell biology of diabetic complications. Nature 414: 813-820, 2001.

11. Arora MK and Singh UK: Molecular mechanisms in the pathogenesis of diabetic nephropathy: An update. Vascul Pharmacol 58: 259-271, 2013.

12. Parving HH, Smidt UM, Friisberg B, Bonnevie-Nielsen V and Andersen AR: A prospective study of glomerular filtration rate and arterial blood pressure in insulin-dependent diabetics with diabetic nephropathy. Diabetologia 20: 457-461, 1981.

13. Maclsaac RJ, Ekinici E and Jerums G: Progressive diabetic nephropathy. How useful is microalbuminuria? Contra'. Kidney Int 86: 50-57, 2014.

14. Glassock RJ: Is the presence of microalbuminuria a relevant marker of kidney disease? Curr Hypertens Rep 12: 364-368, 2010.

15. Rigalleau V, Lasseur C, Raffaitin C, Beauvieux MC, Barthe N, Chauveau $\mathrm{P}$, Combe $\mathrm{C}$ and $\mathrm{Gin} \mathrm{H}$ : Normoalbuminuric renal-insufficient diabetic patients. Diabetes Care 30: 2034-2039, 2007.

16. Clay-Montier LL, Deng JJ and Bai Y: Number matters: Control of mammalian mitochondrial DNA copy number. J Genet Genomics 36: 125-131, 2009.

17. Santos JH, Hunakova L, Chen Y, Bortner C and Van Houten B: Cell sorting experiments link persistent mitochondrial DNA damage with loss of mitochondrial membrane potential and apoptotic cell death. J Biol Chem 278: 1728-1734, 2003.

18. Lee HC and Wei YH: Mitochondrial biogenesis and mitochondrial DNA maintenance of mammalian cells under oxidative stress. Int J Biochemy and Cell Biol 37: 822-834, 2005.

19. Al-Kafaji G and Golbahar J: High glucose-induced oxidative stress increases the copy number of mitochondrial DNA in human mesangial cells. Biomed Res Int 2013: 754946, 2013.

20. Al-Kafaji G, Sabry MA and Bakhiet M: Increased expression of mitochondrial DNA-encoded genes in human renal mesangial cells in response to high glucose-induced reactive oxygen species. Mol Med Rep 13: 1774-1780, 2016.

21. Al-Kafaji G, Sabry MA and Skrypnyk C: Time-course effect of high glucose-induced reactive oxygen species on mitochondrial biogenesis and function in human renal mesangial cells. Cell Biol Int 40: 36-48, 2016.

22. Lee H, Song JH, Shine CS, Park DJ, Park KS, Lee KU and Koh CS: Decreased mitochondrial DNA content in peripheral blood precedes the development of non-insulin-dependent diabetes mellitus. Diabetes Res Clin Pract 42: 161-167, 1998.

23. Gianotti TF, Sookoian S, Dieuzeide G, Garcia S, Gemma C, González CD and Pirola CJ: A decreased mitochondrial DNA content is related to insulin resistance in adolescents. Obesity (Silver Spring) 16: 1591-1595, 2008.

24. Song J, Oh JY, Sung YA, Pak YK, Park KS and Lee HK: Peripheral blood mitochondrial DNA content is related to insulin sensitivity in offspring of type 2 diabetic patients. Diabetes Care 24: 865-869, 2001

25. Xu FX, Zhou X, Shen F, Pang R and Liu SM: Decreased peripheral blood mitochondrial DNA content is related to HbA1c, fasting plasma glucose level and age of onset in type 2 diabetes mellitus. Diabet Med 29: e47-e54, 2012.

26. Hu L, Yao X and Shen Y: Altered mitochondrial DNA copy number contributes to human cancer risk: Evidence from an updated meta-analysis. Sci Rep 6: 35859, 2016.

27. Liu LP, Cheng K, Ning MA, Li HH, Wang HC, Li F, Chen SY, Qu FL and Guo WY: Association between peripheral blood cells mitochondrial DNA content and severity of coronary heart disease. Atherosclerosis 261: 105-110, 2017.

28. Alberti KG and Zimmet PZ: Definition, diagnosis and classification of diabetes mellitus and its complications. Part 1: Diagnosis and classification of diabetes mellitus provisional report of a WHO consultation. Diabet Med 15: 539-553, 1998.

29. Mattix HJ, Hsu CY, Shaykevich S and Curhan G: Use of the albumin/creatinine ratio to detect microalbuminuria: Implications of sex and race. J Am Soc Nephrol 13: 1034-1039, 2002.

30. Stoves J, Lindley EJ, Barnfield MC, Burniston MT and Newstead CG: MDRD equation estimates of glomerular filtration rate in potential living kidney donors and renal transplant recipients with impaired graft function. Nephrol Dial Transplant 17: 2036-2037, 2002. 
31. Lee JE, Park H, Ju YS, Kwak M, Kim J, Oh HY and Seo JS Higher mitochondrial DNA copy number is associated with lower prevalence of microalbuminuria. Exp Mol Med 41: 253-258, 2009

32. Ide T, Tsutsui H, Hayashidani S, Kang D, Suematsu N, Nakamura K, Utsumi H, Hamasaki N and Takeshita A: Mitochondrial DNA damage and dysfunction associated with oxidative stress in failing hearts after myocardial infarction. Circ Res 88: 529-535, 2001.

33. Catherwood MA, Powell LA, Anderson P, McMaster D, Sharpe PC and Trimble ER: Glucose-induced oxidative stress in mesangial cells. Kidney Int 61: 599-608, 2002.

34. $\mathrm{Ha} \mathrm{H}$ and Lee HB: Reactive oxygen species amplify glucose signalling in renal cells cultured under high glucose and in diabetic kidney. Nephrology (Carlton) 10 (Suppl): S7-S10, 2005.

35. Kakimoto $M$, Inoguchi $T$ Sonta $T$, Yu HY, Imamura M, Etoh T, Hashimoto T and Nawata H: Accumulation of 8-hydroxy-2deoxyguanosine and mitochondrial DNA deletion in kidney of diabetic rats. Diabetes 51: 1588-1595, 2002

36. Pan HZ, Zhang L, Guo MY, Sui H, Li H, Wu WH, Qu NQ, Liang MH and Chang D: The oxidative stress status in diabetes mellitus and diabetic nephropathy. Acta Diabetol 47 (Suppl 1) S71-S76, 2010

37. Inci A, Olmaz R, Sarı F, Coban M, Ellidag HY and Sarıkaya M: Increased oxidative stress in diabetic nephropathy and its relationship with soluble Klotho levels. Hiprokratia 20: 198-203, 2016.

38. Zhou M, Zhu L, Cui X, Feng L, Zhao X, He S, Ping F, Li W and $\mathrm{Li}$ Y: Reduced peripheral blood mtDNA content is associated with impaired glucose-stimulated islet $\beta$ cell function in a Chinese population with different degrees of glucose tolerance. Diabetes Metab Res Rev 32: 768-774, 2016.

39. Duran-Salgado MB and Rubio-Guerra AF: Diabetic nephropathy and inflammation. World J Diabetes 5: 393-398, 2014.

40. Cherry AD and Piantadosi CA: Regulation of mitochondrial biogenesis and its intersection with inflammatory responses. Antioxid Redox Signal 22: 965-976, 2015.

41. Chawla A, Nguyen KD and Goh YP: Macrophage-mediated inflammation in metabolic disease. Nat Rev Immunol 11: 738-749, 2011.

42. Wu IC, Lin CC, Liu CS, Hus CC, Chen CY and Hsiung CA: Interrelations between mitochondrial DNA copy number and inflammation in older adults. J Gerontol A Biol Sci Med Sci 72: 937-944, 2017.

43. Cossarizza A: Tests for mitochondrial function and DNA: Potentials and pitfalls. Curr Opin Infect Dis 16: 5-10, 2003.
44. Hurtado-Roca Y, Ledesma M, Gonzalez-Lazaro M, Moreno-Loshuertos R, Fernandez-Silva P, Enriquez JA and Laclaustra M: Adjusting MtDNA quantification in whole blood for peripheral blood platelet and leukocyte counts. PLoS One 11: e0163770, 2016.

45. Steinberg K, Beck J, Nickerson D, Garcia-Closas M, Gallagher M, Caggana M, Reid Y, Cosentino M, Ji J, Johnson D, et al: DNA banking for epidemiologic studies: A review of current practices. Epidemiology 13: 246-254, 2002.

46. Xia P, An H-X, Dang C-X, Radpour R, Kohler C, Fokas E, Engenhart-Cabillic R, Holzgreve W and Zhong XY: Decreased mitochondrial DNA content in blood samples of patients with stage I breast cancer. BMC Cancer 9: 454, 2009.

47. Demmer RT, Zuk AM, Rosenbaum M and Desvarieux M: Prevalence of diagnosed and undiagnosed type 2 diabetes mellitus among US adolescents: Results from the continuous NHANES, 1999-2010. Am J Epidemiol 178: 1106-1113, 2013

48. Gedebjerg A, Almdal TP, Berencsi K, Rungby J, Nielsen JS, Witte DR, Friborg S, Brandslund I, Vaag A, Beck-Nielsen H, et al: Prevalence of micro- and macrovascular diabetes complications at time of type 2 diabetes diagnosis and associated clinical characteristics: A cross-sectional baseline study of 6,958 patients in the Danish DD2 cohort. J Diabetes Complications 32: 34-40, 2018.

49. Cho SB, Koh I, Nam HY, Jeon JP, Lee HK and Han BG: Mitochondrial DNA copy number augments performance of A1C and oral glucose tolerance testing in the prediction of type 2 diabetes. Sci Rep 7: 43203, 2017.

50. Rao M, Li L, Demello C, Guo D, Jaber BL, Pereira BJ and Balakrishnan VS; HEMO Study Group: Mitochondrial DNA injury and mortality in hemodialysis patients. J Am Sco Nephrol 20: 189-196, 2009.

51. Mishra M, Lillvis J, Seyoum B and Kowluru RA: Peripheral blood mitochondrial DNA damage as a potential noninvasive biomarker of diabetic retinopathy. Invest Ophthalmol Vis Sci 57: 4035-4044, 2016

52. Jayakumar RV: Risk factors in diabetic nephropathy. Int J Diabetes Dev Ctries 32: 1-3, 2012.

53. Unsal A, Koc Y, Basturk T, Akgun AO, Sakaci T and Ahbap E: Risk factors for progression of renal disease in patient with diabetic nephropathy. Eur Rev Med Pharmacol Sci 16: 878-883, 2012. 